\title{
artigo
}

Nascimento, E.P.L.; Vizelli, C.; Mariano, R.C.Z; Romano, T.C.R.;

Dimensionamento como dispositivo de fortalecimento e consolidação da Estratégia de Saúde da Família no município Campinas

\section{Dimensionamento como dispositivo de fortalecimento e consolidação da Estratégia de Saúde da Família no município Campinas}

\author{
Dimensioning as a device for strengthening and consolidating the Family Health Strategy in Campinas \\ Dimensionar como dispositivo para fortalecer y consolidar la Estrategia de Salud Familiar en las Campinas
}

\section{RESUMO}

Objetivo: Este estudo teve como objetivo a reelaboração do dimensionamento a partir da revisão da adstrição da clientela por Unidade Básica de Saúde, por faixa etária e sexo, e a definição da população que deveria ser assistida em cada um desses serviços. Método: Para a realização do estudo realizou-se reuniões sistemáticas com coordenadores das áreas técnicas do Departamento de Saúde, apoiadores distritais, gerentes das Unidades Básicas de Saúde e trabalhadores do Departamento de Gestão do Trabalho e Educação na Saúde. Resultados: 0 estudo foi desenvolvido na dimensão qualitativa, com a descrição dos processos de trabalho e da carteira de serviços, e ainda quantitativa, em que apresentamos os cálculos por categoria profissional. Conclusão: Permitiu novos encontros e novas possibilidades de transformação a partir de uma análise situacional, havendo adequação da adstrição de usuários e definição de alocação dos profissionais nas Unidades Básicas de Saúde e nas equipes de Saúde da Família.

DESCRITORES: Dimensionamento; Saúde Pública; Estratégia Saúde da Família; Sistema Único de Saúde.

\section{ABSTRACT}

Objective: This study aimed to re-elaborate the dimensioning from the review of the adstrition of the clientele by Basic Health Unit, by age group and gender, and the definition of the population that should be assisted in each of these services. Method: To carry out the study, systematic meetings were held with coordinators of the technical areas of the Department of Health, district supporters, managers of the Basic Health Units and workers of the Department of Work Management and Health Education. Results: The study was developed in the qualitative dimension, with the description of work processes and the service portfolio, and also quantitative, in which we present the calculations by professional category. Conclusion: It allowed new meetings and new possibilities of transformation from a situational analysis, with adequacy of user adstrition and definition of allocation of professionals in basic health units and health teams of The Family.

DESCRIPTORS: Sizing; Public Health; Family Health Strategy; Unified Health System.

\section{RESUMEN}

Objetivo: Este estudio tenía como objetivo re-elaborar el dimensionamiento de la revisión de la adstrición de la clientela por parte de la Unidad Básica de Salud, por grupo de edad y género, y la definición de la población que debe ser asistida en cada uno de estos servicios. Método: Para llevar a cabo el estudio, se celebraron reuniones sistemáticas con coordinadores de las áreas técnicas del Departamento de Salud, simpatizantes de distrito, gerentes de las Unidades Básicas de Salud y trabajadores del Departamento de Gestión del Trabajo y Educación para la Salud. Resultados: El estudio se desarrolló en la dimensión cualitativa, con la descripción de los procesos de trabajo y la cartera de servicios, y también cuantitativo, en el que presentamos los cálculos por categoría profesional. Conclusión: Permitió nuevas reuniones y nuevas posibilidades de transformación a partir de un análisis situacional, con adecuación de la adstrición de usuarios y definición de asignación de profesionales en unidades básicas de salud y equipos de salud de La Familia.

DESCRIPTORES: Tamaño; Salud Pública; Estrategia de Salud Familiar; Sistema unificado de salud.

RECEBIDO EM: 11/11/2020 APROVADO EM: 01/12/2020 


\section{Elisabet Pereira Lelo Nascimento}

Enfermeira Sanitarista, Mestre em Enfermagem, Doutora em Saúde Coletiva. Apoio Técnico Departamento de Gestão do Trabalho e Educação na Saúde/Secretaria Municipal de Saúde de Campinas.

ORCID: 0000-0001-86496649

\section{Chaúla Vizelli}

Enfermeira Especialista em Gestão em Saúde da Família e Gestão da Clínica Preceptoria. Apoiadora Distrito de Saúde Norte/ Departamento de Saúde/Secretaria Municipal de Saúde de Campinas.

ORCID: 0000-0002-7117-4011

\section{Renata Cauzzo Zingra Mariano}

Enfermeira Especialista Gestão em Serviços de Saúde. Coordenadora da área da Enfermagem Departamento de Saúde/Secretaria Municipal de Saúde de Campinas.

ORCID: 0000-0002-7427-1829

\section{Talita Carlos Rodrigues Romano}

Enfermeira Especializanda em Saúde Pública. Apoiadora Distrito de Saúde Sudoeste/Departamento de Saúde/Secretaria Municipal de Saúde de Campinas.

ORCID: 0000-0001-5382-1345

\section{INTRODUÇÃO}

0 Programa Saúde da Família (PSF) surgiu, no ano de 1994, para transformar a atenção à saúde e reorientar o modelo assistencial, com enfoque na promoção da saúde e prevenção de doenças, de forma a reorganizar os serviços, segundo os princípios de universalidade, integralidade e equidade do Sistema Único de Saúde (SUS)․․ Em 2006, o PSF passou a ser denominado de Estratégia Saúde da Família (ESF). Desde então, a ESF tem alcançado elevada cobertura populacional no atendimento integral da população no Brasil, em 2006 e 2020, suas coberturas foram de $45,3 \%$ e $65,36 \%$ respectivamente 2 .

O município de Campinas, que possui cerca de 1,2 milhão de habitantes, é pólo regional e de referência para cerca de 3,5 milhões de pessoas. Atualmente, de acordo com o Plano Municipal de Saúde da Secretaria Municipal de Saúde (SMS), aproximadamente $55 \%$ da população necessitam de todas as ações assistenciais do Sistema Único de Saúde, no entanto, o dimensionamento foi elaborado para atender $100 \%$ das ações de saúde coletiva. Em 2016, a cobertura assistencial da ESF correspondia a $49,22 \%$. A meta é expandir 5\% desta cobertura, ao ano, entre 2018 a 2021, com a Atenção Primária em Saúde (APS) como ordenadora da atenção à saúde e coordenadora do cuidado, favorecendo a capacidade resolutiva, o potencial de ofertas de ações e serviços, os processos de territorialização e a regionalização em saúde 3 .

Campinas experimentou, ao longo dos anos, diversos arranjos assistenciais na construção do sistema de saúde, destacando-se na implantação do SUS nacionalmente. Em 2001, implantou o PSF-Paidéia, buscando adequar o PSF em um município com características metropolitanas e rede de assistência à saúde multifacetada. Foi um desafio para toda saúde, pois se tratava de agregar as diretrizes do PSF à realidade de uma rede que contava com grande número de especialistas e de não desmantelar conquistas históricas $\mathrm{e}$ consolidadas do sistema local ${ }^{4}$. No entanto, dentre outros determinantes e em decorrência das trocas do governo municipal, da rotatividade de servidores e da insuficiência de médicos generalistas, não ocorreu a consolidação desse modelo de saúde no município.

Nesse contexto, a SMS iniciou, por meio do Departamento de Gestão do Trabalho e Educação na Saúde (DGTES), em 2006, um estudo sobre dimensionamento da força de trabalho na APS com intuito de solucionar os problemas mais frequentes vivenciados na gestão do trabalho e tentar qualificar a assistência aos usuários. Em 2013, essa experiência foi selecionada pelo Prêmio InovaSUS, valorização de boas práticas e inovação na saúde, promovido pelo Ministério da Saúde, na Secretaria de Gestão do Trabalho e da Educação na Saúde, por meio do Departamento de Gestão do Trabalho em Saúde (DEGTS). Foi uma das experiências analisadas para o desenvolvimento da metodologia de avaliação de boas práticas em gestão do trabalho no SUS pela Escola Andaluzia de Saúde Pública/Espanha e pelo Laboratório de Inovação da Gestão do Trabalho na Saúde, criado pelo DEGTS ${ }^{5,6}$.

O presente estudo teve como objetivo a reelaboração do dimensionamento a partir da revisão da adstrição da clientela por UBS, por faixa etária e sexo, e a definição da população que deveria ser assistida em cada um desses serviços, considerando os seguintes princípios do SUS: regionalização, equidade, universalidade e integralidade. Portanto, fez-se necessário também a reavaliação das vulnerabilidades sociais dessa população, sendo mantidos os indicadores de moradia, escolaridade e renda.

\section{MÉTODO}

Em 2017, a SMS, com objetivo de contribuir para a construção de um SUS mais eficiente e promotor de inovações na busca da equidade e do respeito às necessidades da população, adotou o modelo assistencial da ESF. Nessa perspectiva, iniciou-se a revisão deste trabalho de dimen- 


\section{artigo}

Nascimento, E.P.L.; Vizelli, C.; Mariano, R.C.Z.; Romano, T.C.R.;

Dimensionamento como dispositivo de fortalecimento e consolidação da Estratégia de Saúde da Família no município Campinas

sionamento da força de trabalho na APS como um dispositivo de fortalecimento e consolidação da ESF, em um município de grande porte, com uma rede de serviços de saúde estruturada e de alta complexidade. Discorreremos a metodologia desenvolvida na dimensão qualitativa, com a descrição dos processos de trabalho e a carteira de serviços, e, após, a quantitativa, em que explicitaremos os cálculos por categoria profissional. Destacamos que a alocação dos profissionais nas equipes de Saúde da Família (eSF) e por Unidade Básica de Saúde (UBS) inclui dados de vulnerabilidade dos territórios e de necessidades de saúde dos usuários.

Por meio de reuniões sistemáticas com coordenadores das áreas técnicas do Departamento de Saúde, apoiadores distritais, gerentes das UBS e trabalhadores do DGTES, foram realizados novos estudos e novas análises das diversas áreas e categorias profissionais, em que foram redefinidos parâmetros e indicadores para a quantificação da força de trabalho em saúde. Ressaltamos que a participação de gerentes das UBSs qualificou, de forma relevante, todo o processo, na medida em que socializavam o cotidiano das UBSs, as experiências vivenciadas na assistência e as dificuldades com o dimensionamento vigente para a consolidação da ESF.

Foram utilizadas questões norteadoras para análise situacional e reelaboração do dimensionamento: Para quem? Usuários nos territórios onde vivem e suas necessidades de saúde. O que oferecer? Serviços instalados que atendam às necessidades de saúde dos usuários. Como? O processo organizativo dos serviços de saúde, onde são desenvolvidas as atividades de promoção, prevenção e recuperação da saúde. Por fim, quantos? Os profissionais necessários para prestar assistência qualificada e resolutiva aos usuários.

As questões - Para quem? O quê? Como? Quantos? - permitiram novas reflexões e novos desdobramentos. Foram analisados quem eram os usuários que viviam no território, considerando a vulnerabilidade social e os indicadores epidemiológicos. Isso resultou na reorganização

dos processos de produção de serviços, com a finalidade de atender às necessidades de saúde dos grupos sociais, promo-

Para o cargo de médico (Medicina de Família e

Comunidade), foi dimensionado um profissional de

\section{6 horas por eSF,}

variando a adstrição

de usuários por

equipe de acordo

com o grau de

vulnerabilidade.

Tal resultado foi

decorrente da

multiplicação do

número de $\mathrm{eSF}$ por

um profissional de

36 horas. vendo atenção à saúde mais humanizada e qualificada. Houve, também, a adequação da carteira de serviços ofertada na APS. Posteriormente a todas as definições e todos os reordenamentos, foram realizados cálculos matemáticos, que resultaram na identificação da quantidade de profissionais necessários para assistir os usuários com qualidade ${ }^{7}$.

\section{RESULTADOS}

Este estudo determinou o número de usuários por eSF, sendo que, para as áreas de baixa vulnerabilidade, a adstrição foi de 4.000 habitantes por eSF; de média vulnerabilidade, 3.500 habitantes por eSF; de alta vulnerabilidade, 3.000 habitantes por eSF e, para áreas rurais, 2.000 habitantes por eSF. Ao dividirmos o quantitativo total da população adstrita a um determinado território pelo quantitativo populacional de acordo com as vulnerabilidades sociais, obtivemos o número necessário de eSF para cada $\mathrm{UBS}^{8}$. Esse resultado fundamentou o dimensionamento de todas as categorias profissionais que desenvolvem suas ações na APS, em linha com a diretriz da SMS de ampliar a cobertura da ESF no município de Campinas.

Para o cargo de médico (Medicina de Família e Comunidade), foi dimensionado um profissional de 36 horas por eSF, variando a adstrição de usuários por equipe de acordo com o grau de vulnerabilidade. Tal resultado foi decorrente da multiplicação do número de eSF por um profissional de 36 horas. Do total da carga horária semanal, subtraiu-se $20 \%$ para atividades educativas, participação em reuniões e visitas domiciliares. Os $80 \%$ da jornada estavam distribuídos entre consultas programáticas e de agudos, variando de três a quatro atendimentos por hora

O profissional enfermeiro foi dimensionado um servidor de 36 horas por eSF, utilizando-se os mesmos parâmetros do cargo de médico. No entanto, a divisão da jornada semanal estava distribuída entre 20\% para ações educativas e reuniões, $20 \%$ para ações administrativas e de supervisão a equipe de enfermagem e $60 \%$ para con- 
sultas programáticas e de acolhimento aos usuários, prevendo duas a quatro consultas por hora ${ }^{8,9}$.

O cálculo de técnico em enfermagem foi realizado a partir dos horários de funcionamento das UBSs e de retaguarda assistencial, de modo que, quando o funcionamento foi inferior a 60 horas semanais, foram dimensionados dois técnicos em enfermagem por eSF e mais quatro profissionais de retaguarda. As UBSs com funcionamento igual a 60 horas semanais foram dimensionadas com três técnicos em enfermagem por eSF e mais cinco profissionais de retaguarda. Já as UBSs com funcionamento maior que 60 horas semanais foram dimensionadas com três técnicos em enfermagem por eSF e mais seis profissionais de retaguarda. A distribuição da jornada ficou com 20\% para atividades administrativas e reuniões, $20 \%$ para participação em ações educativas e 60\% para assistência individual, sendo a média de quatro atendimentos ou procedimentos por hora.

Para o emprego de Agente Comunitário de Saúde (ACS), foi considerada a média de 750 habitantes por profissional, com a possibilidade do gerente da UBS estratificar os usuários adstritos por ACS a partir da classificação de risco social, epidemiológico e conforme a vulnerabilidade dos microterritórios. Para essa categoria, estabeleceu-se que $20 \%$ da carga horária semanal seriam utilizadas para ações educativas, administrativas e reuniôes e $80 \%$ para visitas domiciliares, com a meta de realizar uma visita familiar por hora, cadastrando e acompanhando a situação de saúde desses usuários ${ }^{8}$.

Para a equipe de saúde bucal (dentista e auxiliar de saúde bucal), foi considerada a capacidade física instalada. $O$ cálculo utilizou o número de cadeiras odontológicas existentes multiplicadas pelo horário de funcionamento da UBS, dividido pela carga horária dos profissionais de 36 horas. Deste número, consideramos 15\% do tempo para higienização, desinfecção e ações educativas. Ainda, conforme a meta de cobertura de eSF estabelecida, a capacidade física é avaliada e, quando pertinente, é indicada a necessidade de ampliação. $\mathrm{O}$ cálculo utilizado foi o mesmo para os dentistas e auxiliares de saúde bucal por se tra-

Para provimento

do quantitativo de profissionais

necessários à

recomposição das

equipes de Saúde

da Família, o

município realizou

concursos públicos

no mês de julho

de 2019 para os

respectivos cargos:

Auxiliar em Saúde

Bucal, Dentista,

Enfermeiro,

Médico de Família e

Comunidade. tar de um trabalho desenvolvido a quatro mãos, durante a assistência odontológica ao usuário.

O cargo de farmacêutico foi dimensionado para atuar nos Núcleos Ampliados de Saúde da Família (Nasf), vinculados em uma no mínimo e nove eSF no máximo. A atuação do farmacêutico foi organizada em 50\% da jornada para atendimentos individuais na unidade de saúde e no domicílio, 20\% para gerenciamento e 30\% para ações educativas e matriciamentos aos profissionais das UBSs.

O agente de apoio à saúde (Farmácia) foi calculado a partir da maior média de receitas dispensadas nos últimos cinco anos, por UBS, dividido pela capacidade produtiva desse profissional. De um profissional com carga horária de 36 horas, subtraiu-se $15 \%$ para atividades de armazenamento, controle de estoque e ações educativas. $\mathrm{O}$ restante das horas foram multiplicadas pela média de 12 receitas atendidas por hora e, por fim, multiplicou-se pela média de semanas do ano.

\section{DISCUSSÃO}

Para provimento do quantitativo de profissionais necessários à recomposição das equipes de Saúde da Família, o município realizou concursos públicos no mês de julho de 2019 para os respectivos cargos: Auxiliar em Saúde Bucal, Dentista, Enfermeiro, Médico de Família e Comunidade. Os profissionais aprovados já estão sendo convocados. A respeito dos cargos de Agente Comunitário de Saúde e Técnico de Enfermagem, no momento da publicação de tal edital, encontrava-se vigente um concurso anterior com candidatos classificados, os quais foram convocados para tomar posse das vagas existentes.

Todos os profissionais que compõem a eSF devem oferecer, à população, de forma uniforme, um padrão de serviços essenciais nas unidades de saúde da família, chamado de carteira de serviços. A SAPS/MS lançou a carteira de serviços da Atenção Primária à Saúde (CaSAPS) em 18 de dezembro de 2019. Trata-se de uma lista para orientar sobre as ações e os serviços clíni- 


\section{artigo}

Nascimento, E.P.L.; Vizelli, C.; Mariano, R.C.Z.; Romano, T.C.R.;

Dimensionamento como dispositivo de fortalecimento e consolidação da Estratégia de Saúde da Família no município Campinas

cos e de vigilância em saúde que podem ser ofertados na APS e será norteadora das ações de saúde, com atuação multiprofissional e sendo orientadora dos serviços ${ }^{10}$. No município de Campinas, a carteira de serviços oferecida à população atualmente possui: consultas em enfermagem, clínica médica, odontologia, pediatria, ginecologia e obstetrícia, serviços assistenciais - como dispensação de medicamentos; vacinação de adultos e crianças; exames laboratoriais; biópsias; eletrocardiograma; vigilância em saúde; dentre outros ${ }^{11}$.

A transparência no conjunto de ações oferecidas permite, aos usuários, fiscalizar, avaliar e qualificar a APS. Portanto, observamos que a carteira de serviços, assim como o dimensionamento, pode constituir-se como uma importante ferramenta de gestão.

Vários desafios estavam postos na elaboração desta proposta. Dentre eles, destacam-se: a escassez de uma literatura específica sobre dimensionamento na Saúde Pública, especificamente na Atenção Primária à Saúde; a necessidade de revisão e atualização dos parâmetros e indicadores epidemiológicos e sociais para reclassificar as vulnerabilidades das UBS; a alteração dos mapas territoriais, modificando a adstrição de usuários por UBS; a modernização do sistema de informação, que possibilitasse o acesso local e a atualização permanente de recursos humanos; e, por fim, o número reduzido de registros dos atendimentos e das consultas realizadas pelos profissionais, que causavam distorção nos relatórios de produção ambulatorial.

No âmbito da SMS, várias ações estão sendo realizadas com o objetivo de expansão e fortalecimento da Estratégia de Saúde da Família: realização de concurso público para vários cargos, para recomposição e ampliação das eSF e dos Nasf; implantação do Programa Mais Médicos Campineiro - que objetiva a formação de médicos da saúde da família e comunidade; implantação da residência multiprofissional em enfermagem e odontologia; e desenvolvimento de processos de capacitação e de educação permanente com a valorização do trabalho multi e interprofissional.

Todas essas ações ocorrem no sentido de aperfeiçoar a atenção à saúde nos vá- rios ciclos de vida, com a implantação de processos relativos à gestão do acesso, da agenda e do território, além da revisão e inovação de protocolos assistenciais. A meta era cadastrar $80 \%$ da população do município até abril de 2020, no entanto, devido à pandemia do Covid-19, esse propósito ainda não foi atingido.

\section{CONCLUSÃO}

A adequação do método de dimensionamento em prol da reorganização do modelo assistencial no município de Campinas permitiu novos encontros e novas possibilidades de transformação a partir de uma análise situacional, havendo adequação da adstrição de usuários e definição de alocação dos profissionais nas Unidades Básicas de Saúde e nas equipes de Saúde da Família. Atualmente, está em pauta a possibilidade de expansão do sistema de informação para garantir a eficiência e a eficácia dos dados e das informações. Ressaltamos, ainda, que esse dispositivo tem qualificado as negociações entre os gestores municipais na definição orçamentária relacionada às necessidades de contratações.

\section{REFERÊNCIAS}

1. Nascimento EPL, Correa CBS. O agente comunitário de saúde: formação, inserção e práticas. Cad. Saúde Pública. Junho de 2008. Vol. 24 (6): 1304-1313.

2. Ministério da Saúde (Brasil). Secretaria de Atenção Primária à Saúde. Departamento de Atenção Básica. Informação e gestão da Atenção Básica. E-gestor. Brasilia: Ministério da Saúde,2020. [Acesso em 24 de junho de 2020]. Disponível em: https://egestorab. saude.gov.br/paginas/acessoPublico/relatorios/relHistoricoCoberturaAB.xhtml..

3. Campinas. (Município). Secretaria de Saúde. Departamento de Gestão e Desenvolvimento Organizacional. Campinas, 2018. [acesso em 2019 dez 4] Disponivel em: http://www.campinas.sp.gov.br/ saude.

4. Campos GWS. Saúde Paidéia. São Paulo, Brasil: Hucitec Editora; 2003.

5. Ministério da Saúde (Brasil). Secretaria de Gestão do Trabalho e da Educação na Saúde. Departamento de Gestão e de Regulação do Trabalho na Saúde. Prêmio InovaSUS 2012/2013: valorização das práticas e inovação na gestão do trabalho na saúde. Brasilia: Ministério da Saúde, 2015.

6. Pinzón S, Herrera M, Mena AL. Boletín. Visita in loco: Metod- ología análisis de buenas prácticas en gestión del trabajo en el SUS. Campinas, 2015.

7. Nascimento EPL, Carmona SAMLD. A experiência da elaboração do dimensionamento na atenção Básica da Secretaria Municipal de Saúde de Campinas. Rede Unida. No prelo 2020.

8. Brasil. Ministério da Saúde. Portaria n².436, de 21 de setembro de 2017. Aprova a Política Nacional de Atenção Básica (PNAB) [estabelecendo a revisão de diretrizes para a organiza ^ção da Atenção Básica, no âmbito do Sistema Único de Saúde (SUS)]. Diário Oficial[da], República Federativa do Brasil. 2017 set. 183; Seção 1.p 68.

9. Brasil. Ministério da Saúde. Portaria n 1.631 de outubro de 2015. Dispõe sobre critérios e parâmetros para a programação de ações e serviços de saúde no âmbito do Sistema Único de Saúde. Diário Oficial [da] República Federativa do Brasil. 2015 out.

10. Ministério da Saúde (BR). Secretaria de Atenção Primária. Carteira de serviços da atenção primária à saúde (CaSAPS). Versão: profissionais de saúde e gestores (resumida). Brasília: Ministério da Saúde, 2019.

11. Campinas. (Município). Secretaria de Saúde. Departamento de Saúde. Campinas, 2018 [acesso em 2019 dez 17] Disponível em: http://www.campinas.sp.gov.br/saude. 